\title{
Outpatient evaluation of oxidative stress in subjects undergoing systemic oxygen-ozone therapy
}

\author{
Fortunato Loprete, ${ }^{1,2}$ Francesco Vaiano, ${ }^{2,3}$ Luigi Valdenassi ${ }^{2,4}$ \\ ${ }^{1}$ Private practitioner, Surgeon, Padova; ${ }^{2}$ Italian Society for Oxygen-Ozone Therapy (SIOOT), Gorle (BG); ${ }^{3}$ Private \\ practitioner, Surgeon, Desio (MB); ${ }^{4}$ University of Pavia, Pavia, Italy
}

\begin{abstract}
Oxygen-ozone therapy improves the quality of life in patients affected by various diseases.

The purpose of this study was to verify and eventually quantify the production of free radicals generated by oxidative stress induced by the ozonated auto-hemoinfusion in subjects subjected to this practice and eventually evaluate and naturally quantify the plasma antioxidant power of the subjects under examination. All subjects showed a significant improvement in the oxidative balance already at the first check, which was maintained (albeit with a minimal non-significant loss) at the end of the study.

The results of the study have made it possible to verify that systemic ozonated auto-hemoinfusion not only does not involve further oxidative stress but even exerts an important antioxidant effect, through the scavenger effect of free radicals.
\end{abstract}

\section{Introduction}

When we talk about oxygen-ozone therapy, we are referring to a therapy that uses a mixture of oxygen $\left(\mathrm{O}_{2}\right)$ and ozone $\left(\mathrm{O}_{3}\right)$ : in medi-

\footnotetext{
Correspondence: Fortunato Loprete, Italian Society for Oxygen-Ozone Therapy (SIOOT), via Don Luigi Sturzo 2, 24020 Gorle (BG), Italy.

E-mail: info@ossigenoozono.it
}

Key words: Oxidative stress; Oxygen-ozone therapy; Oxygenation; Antioxidation; Disinfection.

Contributions: the authors contributed equally.

Conflict of interest: the authors declare no potential conflict of interest.

Funding: none.

Received for publication: 1 March 2019

Accepted for publication: 14 March 2019.

CCopyright F. Loprete et al., 2019

Licensee PAGEPress, Italy

Ozone Therapy 2019; 4:8175

doi:10.4081/ozone.2019.8175

This article is distributed under the terms of the Creative Commons Attribution Noncommercial License (by-nc 4.0) which permits any noncommercial use, distribution, and reproduction in any medium, provided the original author(s) and source are credited. cine, ozone is never used pure, yet always mixed in a small percentage, about $3 \%$, with the medical oxygen that acts as a vehicle.

Remaining within the therapeutic range, as a concentration, as indicated in the protocols, ozone has no toxic effect but becomes an extraordinarily versatile therapeutic agent; it improves, in fact, the quality of life in patients affected by pathologies such as: chronic fatigue, chronic venous insufficiency of the lower limbs, inflammatory osteo-articular and intestinal diseases, chronic benign and non-benign pain, up to much more important diseases such as multiple sclerosis, advanced life support, Alzheimer's disease, rheumatoid arthritis, Parkinson's disease, early senile dementia, Crohn's disease. ${ }^{1}$

These indications are dictated by the fact that this is a broad spectrum therapy, now recognized and widely tested: anti-inflammatory action, pain-relieving (local and systemic), reactivates the microcirculation in the various organs and systems (greater release and better use of oxygen and nutrients in the cells) and better elimination of toxic substances produced by cellular metabolism.

The biological effects of ozone are manifold. There are three basic mechanisms:

i) Oxygenation: ozone improves the ability of the blood to supply oxygen to the tissues: this results in a reactivation of the microcirculation and peripheral oxygenation.

ii) Antioxidation: ozone, which is an oxidizer in itself, strongly stimulates the reactivation of the systems defensive enzymatic antioxidants of the body, those who must defend it from toxic aggressions and oxidative processes of aging. This explains its effectiveness in all those chronic pathologies in which the oxidative stress is involved, the most nowadays.

iii) Disinfection: it is a very powerful agent against bacteria, fungi, viruses, parasites: practically no microorganism resists the oxidizing action of ozone..$^{2-5}$

Another and not less important therapeutic properties of the ozone therapy: anti-inflammatory and analgesic action, activation of reparative and regenerative processes, blood fluidification, immuno-stimulation and immuno-modulation, reduction of insulin-resistance and stimulation of detoxification processes.

The purpose of this study was to verify and eventually quantify the production of free radicals generated by oxidative stress induced by the ozonated auto-hemoinfusion in subjects subjected to this practice and eventually evaluate and naturally quantify the plasma antioxidant power of the subjects under examination.

Auto-hemoinfusion perfectly executed (according to the guidelines drawn up by the Italian Society for Oxygen-Ozone Therapy, SIOOT, and filed with the Italian Ministry of Health).

The first part of the self-emergence-infusion is called withdrawal, and as such must follow the directives issued by the Ministry of Health which dictates the following procedure:

i) The sampling must be a fall (physiological). 
ii) The blood must be mixed continuously during the blood collection: use a blood mixer/weigher.

iii) The quantity in $\mathrm{mL}$ must be set before starting the procedure: use a scale with the possibility to set the withdrawal in $\mathrm{mL}$.

iv) At the end of the process, the sampling must be automatically blocked: use a scale with automatic locking.

Only specific SANO3 auto-hemoinfusion bags are used.

Blood is taken from a peripheral vein. The blood is drained into the bag until it reaches the programmed quantity (about 150-200 cc). The quantity is checked by weighing the bag using a tilting scale.

From the ozone generator, the oxygen-ozone mixture is taken at the desired concentration $(20,30,40$ or $50 \mu \mathrm{g} / \mathrm{mL})$ using a $50-$ cc syringe, and it is introduced into the bag through the opening until the dose required by the protocol.

Infusion is carried out at the rate of $60 \sim 80$ drops per minute. The total amount of blood used in the session and the total amount of ozone used are recorded. The total number of sessions can be 10-20. Treatment can be repeated 1-2 times a week.

This method guarantees:

i) Physiological withdrawal by fall with sure benefit for the patient;

ii) Very high quantity of valid red blood cells (absence of foam and hemolysis);

iii) Oxygenation with $\mathrm{O}_{2} \mathrm{O}_{3}$ much more effective for the patient.

The oxidative stress (meant as the imbalance between the generation of so-called free radicals, produced in excess, and the elimination, by the antioxidant defense systems) is considered an emerging risk factor for health that is increasingly confirmed by many scientific evidence. ${ }^{6-10}$

This condition, in fact, is associated not only with aging but also with a number of diseases, such as cardiovascular diseases, neuro-degenerative disorders, metabolic disorders and numerous other chronic-degenerative diseases, including neoplasms.

Often oxidative stress does not present its own symptoms and does not give rise to a characteristic clinical picture, so it can only be highlighted through specific biochemical tests that can highlight the excessive production of free radicals and the deficit of antioxidant defense systems.

Among the various tests currently available for outpatient use on blood samples, the authors have used the FRAS 5 Evolvo system (H\&D srl-Parma). ${ }^{11-14}$

This is precisely the reactive oxygen metabolites (d-ROMs) test (determination of total oxidizing capacity, normal values 250-300 U CARR) and the Plasma Antioxidant Test (PAT) test determination of biological antioxidant potential, optimal values 2200-2800 U Cor), the antioxidant test most used so far in the clinical setting, which allows you to determine more quickly and more accurately the level of antioxidants in the blood.

These tests have rapidly spread in the clinical routine both in our country and in other countries, European and non-European, not only for their excellent analytical performance (sensitivity, specificity, repeatability, etc.) but also for their ease of execution.

The antioxidant capacity of plasma, in fact, is a fundamental measure to understand the ability of our body to deal with the condition of oxidative stress generated by a wide range of endogenous and exogenous factors.

The d-ROMs test is an outpatient photometric test using an analytical instrument called a photometer, which can be performed on whole blood samples (generally capillary, obtained by acupuncture, but also intravenously), serum, heparin plasma and some extracellular fluids.

The d-ROMs test essentially determines the blood concentration of hydroperoxides, substances belonging to the broad class of so-called ROMs.

\section{Materials and Methods}

The objective of the study, which lasted a total of 120 days, was to verify the effects induced by the systemic ozonated auto-hemoinfusion (GAE) on the overall state of well-being, through the evaluation of oxidative stress and the antioxidant system of the 45 total subjects (healthy and with various diseases) enrolled in the study.

At the beginning, at the end of the 15 sessions (precisely 2 days later) and at the end of the study, 60 days after the last GAE, the oxidative balance was evaluated by examining the d-ROMs test and the PAT test (Tables 1 and 2).

\section{Results}

In all the subjects evaluated, there was a slight reduction in the free radicals taken into consideration, from 565.5U CARR the values were reduced to reach values of 402.9 two months after the end of the cycle of auto-hemoinfusion (Normal range: 250-300 U CARR; 1 U CARR corresponds to $0.08 \mathrm{mg} \mathrm{H}_{2} \mathrm{O}_{2} / \mathrm{dL}$ and a statistically significant increase in the antioxidant response, which from the initial values of 2053 increased to an average of 2217.9 (1 U Cor $=1.4$ micromole/L Vitamin C).

\section{Discussion and Conclusions}

All subjects showed a significant improvement in the oxidative balance already at the first check, which was maintained (albeit with a minimal non-significant loss) at the end of the study.

The results of the study have made it possible to verify that

Table 1. Severity of oxidative stress based on d-ROMs test values.

\begin{tabular}{lcc} 
ROM (U CARR) & $\begin{array}{c}\text { ROM } \\
\left(\mathrm{mg} \mathrm{H}_{2} \mathrm{O}_{2} / \mathrm{dL}\right)\end{array}$ & $\begin{array}{c}\text { Oxidative stress } \\
\text { (gravity) }\end{array}$ \\
$300-320$ & $24.08-25.60$ & Border-line condition \\
$321-340$ & $25.68-27.20$ & Mild oxidative stress \\
\hline $341-400$ & $27.28-32.00$ & Average oxidative stress \\
$401-500$ & $32.08-40.00$ & High oxidative stress \\
\hline$>500$ & $>40.00$ & Very high oxidative stress \\
\hline
\end{tabular}

Normal range: 250-300 U CARR. $1 \mathrm{U}$ CARR corresponds to $0.08 \mathrm{mg} \mathrm{H}_{2} \mathrm{O}_{2} / \mathrm{dL}$. ROM, reactive oxygen metabolite.

Table 2. Plasma Antioxidant Test (PAT): reference values. Reference values are expressed in $U$ Cor. In this case the reference antioxidant is Vitamin $\mathrm{C}$, an iron-reducing agent used as standard.

\begin{tabular}{lc}
\hline$>2800$ U Cor & Excess values \\
$2200-2800$ U Cor & Normal values \\
\hline $2000-2000$ U Cor & Minimum borderline limit \\
$2000-1800$ U Cor & State of slight deficiency \\
\hline$<1800$ U Cor & State of deficiency \\
\hline 1 U Cor $=1.4$ micromole/l Vitamin C. & \\
Use of antioxidants and/or cellular damage (endothelial cells, erythrocytic hemolysis), activation of \\
cell reactivity (increase in uric acid and phosphates). \\
$\begin{array}{l}\text { Metrological characteristics. Specificity: the PAT test is specific for the biological potential of systemic } \\
\text { antioxidants. Accuracy: the test is accurate from } 500 \text { up to } 10000 \text { U Cor. }\end{array}$
\end{tabular}


GAE not only does not involve further oxidative stress but even exerts an important antioxidant effect, through the scavenger effect of free radicals.

Based on the results obtained, very encouraging, in terms of improvement of the parameters considered and the lack of undesirable side effects, the use of GAE, both in healthy subjects (for prevention) and in subjects with various diseases, can be considered as a valid approach in the treatment of those subjects who have a significant oxidative stress pathological.

If we consider oxidative stress as a real pathology that can involve the entire regulatory system, such as to underlie the multifactorial pathogenesis of many other diseases, especially chronic ones (allergies, immune deficiency diseases, rheumatic diseases, cardiovascular diseases, headaches, neurosis, etc.), it seems particularly important to consider, both as a preventive measure in apparently healthy subjects and, for therapeutic purposes, in patients suffering from chronic allergic and/or degenerative diseases, the usefulness of ozonated GAE.

\section{References}

1. Vaiano F, Loprete F. Large auto-hemoinfusion versus rectal insufflation in patients with metabolic syndrome. Ozone Ther 2016;1:13-16.

2. Franzini M, Valdenassi L, Ionita G. First evaluations of oxygen-ozone therapy in antibiotic-resistant infections. Ozone Ther 2016;1:5-7.

3. Bocci V, Zanardi I, Huijberts MS, Travagli V. Diabetes and chronic oxidative stress. A perspective based on the possible usefulness of ozone therapy. Diabetes Metab Syndr 2011;5:45-9.

4. Bocci V, Aldinucci C. Biochemical modifications induced in human blood by oxygenation-ozonation. J Biochem Mol Toxicol 2006;20:133-8.

5. Bocci V, Luzzi E, Corradeschi F, et al. Studies on the biological effects of ozone, 3 : an attempt to define conditions for opti- mal induction of cytokines. Lymphokine Cytokine Res 1993;12:121-6.

6. Cornelli U, Cornelli M, Terranova R, et al. Ageing and free radicals [Invecchiamento e radicali liberi]. Prog Nutr 2000;3:37-50.

7. Cornelli U, Cornelli M, Terranova R, et al. The importance of oxidative stress as risk factor for morbidity [Importanza dello stress ossidativo come fattore di rischio per la morbilità]. Med Biol 2000;1:13-8.

8. Cornelli U, Cornelli M, Terranova R. Free radical and vascular diseases. Int Union Angiol Bull 1999;15:7-10.

9. Cornelli U, Terranova R, Luca S, et al. Bioavailability of some food supplementations in men and women using the d-ROMs test as a marker of oxidative stress. J Nutr 2001;131:3208-11.

10. Alberti A. Report of a EPR experiment on Diacron's kit [Esperimento EPR sul kit Diacron]. I.Co.C.E.A. - National Council of Research. Research Area. Bologna, Italy: I.Co.C.E.A.; 1997.

11. Alberti A. The d-ROMs test. Model and chemical basis [Il dROMs test. Modello e basi chimiche]. I.Co.C.E.A. - National Council of Research. Research Area. Bologna, Italy: I.Co.C.E.A.; 1997.

12. Alberti A, Bolognini L, Carratelli M, et al. Assessing oxidative stress with the d-ROMs test. Some mechanistic considerations. Proceedings of the SFRR Europe Summer Meeting. 1997 June 26-28, Abano Terme, Italy. Abano Terme, Italy: SFRR; 1997. pp 82-83.

13. Alberti A, Bolognini L, Macciantelli D, Carratelli M. The radicalcation of N,Ndiethyl-para-phenylendiamine: a possible indicator of oxidative stress in biological samples. Res Chem Intermed 2000;26:253-67.

14. Franzini A, Luraschi P, Pagani A. Direct measurement of reactive oxygen metabolites in blood serum: analytical assesment of a novel method [Misura diretta dei metaboliti reattivi dell'ossigeno nel siero: valutazione analitica di un nuovo metodo]. Biochim Clin 1996;89:5-6. 